\title{
Regulation of bcl-2 gene family members in human endometrium by antiprogestin administration in vivo
}

\author{
H. O. D. Critchley ${ }^{1}$, S. Tong ${ }^{1}$, S. T. Cameron ${ }^{1}$, T. A. Drudy ${ }^{1}$, R. W. Kelly ${ }^{2}$ and D. T. Baird ${ }^{1}$ \\ 'Department of Obstetrics and Gynaecology, The University of Edinburgh, 37 Chalmers Street, Edinburgh EH3 9ET, UK; and \\ ${ }^{2}$ Medical Research Council Reproductive Biology Unit, Centre for Reproductive Biology, 37 Chalmers Street, Edinburgh EH3 9ET, UK
}

It is likely that the changes which occur in the endometrium throughout the menstrual cycle involve apoptosis, and that expression of associated genes, such as the bcl-2 family, are regulated by sex steroids. The aim of this study was to investigate the presence of bcl-2, Bax and oestrogen receptor proteins in secretory endometrium collected from ten patients with normal ovulatory cycles 4 or 6 days after the LH surge, and on the same days in a subsequent cycle in which the formation of secretory changes was inhibited by the administration of the antiprogestin mifepristone (RU486) 2 days after the onset of the LH surge. Since some stromal cells display positive immunoreactivity, leucocyte subpopulations of macrophages (CD68-positive) and large granular lymphocytes (CD56-positive) were identified in serial sections. After administration of mifepristone on day 2 after the LH surge, a significant increase in bcl2 immunoreactivity was observed in glandular and surface epithelium. A positive correlation (0.874) with nuclear oestrogen receptor immunoreactivity in endometrial glands was demonstrated. Subsets of stromal cells, identified as CD68-positive macrophages and CD56-positive large granular lymphocytes displayed positive immunoreactivity for the bcl-2 epitope, which was unaffected by mifepristone administration. Bax immunostaining was similar in control and antiprogestin-treated endometrium. These data indicate that antiprogestin administration inhibits progesterone downregulation of steroid receptors in endometrial glands, resulting in persistence of a proliferative endometrium and accompanying bcl-2 secretion.

\section{Introduction}

The term apoptosis describes the mode of cell death that is responsible for the deletion of cells in normal tissues (Wyllie et al., 1980). The process is distinct from necrosis and displays characteristic features. Regulation of the apoptotic process is complex, and a family of related proteins, the bcl-2 gene family, have either a positive or negative role. bcl-2 protein is the prototype of the gene family that is associated with the inhibition of apoptosis (Wyllie, 1994). One mode of regulation is via the interaction of bcl-2-related apoptosis inhibitors and apoptosis promoters, for example, Bax protein (Oltavi et al., 1993). Bax protein, also a bcl-2 family member, promotes cell death, possibly countering the anti-apoptotic action of bcl-2 through heterodimer interaction (Oltavi et al., 1993).

The role and regulation of apoptotic events in human endometrium remains unknown. Kokawa et al. (1996) observed apoptosis in the functional layer of early proliferative and late secretory phase endometrium, although there was no evidence for apoptosis in the basal layers. These observations were consistent with much earlier

Received 14 July 1998. reports (Hopwood and Levison 1976) of a morphological increase in the number of apoptotic bodies in pre-menstrual and menstrual phase endometrium.

The predominance of bcl-2 in proliferative endometrium was reported by this group (Koh et al., 1995) and confirmed by others (McLaren et al., 1997; Tao et al., 1997). Tao et al. (1997) also described maximal immunoreactivity in the basal endometrium. In contrast, the same group reported that Bax protein immunoreactivity was low in the proliferative phase, but increased markedly in secretory phase endometrial tissues, particularly in the functional epithelial layers. These results support a co-ordinated shift towards greater concentrations of the pro-apoptosis protein Bax in the glandular compartment of the secretory phase. In a study of Bax immunoreactivity in normal endometrium, McLaren $e t$ al. (1997) reported positive Bax immunostaining throughout the menstrual cycle, but did not specify whether this was in the functional or basalis layer. The endometrial tissues were collected by sharp curettage and hence were likely to include elements of the basalis.

It is highly possible that the expression of genes that encode for apoptosis-related proteins are regulated by sex steroid hormones. In this context, Otsuki et al. (1994) briefly reported a correlation between oestrogen and progesterone 
receptor expression and the cyclic variations in bcl-2 in human endometrium in glandular epithelial cells.

Mifepristone is an $11 \beta$ phenyl substituted steroid that is similar in structure to progesterone. The compound lacks the C-19 methyl group and the $2 \mathrm{C}$ side chain at C-17 of progesterone (Baulieu, 1997). Mifepristone binds strongly to the progesterone receptor and glucocorticoid receptor, but does not bind to the oestrogen receptor. Affinity for the glucocorticoid receptor and weak binding for the androgen receptor is not surprising in view of the structural homology among the family of steroid receptors. It has been demonstrated that, in women, mifepristone will induce endometrial bleeding in the luteal phase of the menstrual cycle and abortion in early pregnancy (Baird, 1993). A single dose of $200 \mathrm{mg}$ given to women shortly after ovulation retards the development of a secretory endometrium and a number of progesterone-dependent products (Swahn et al., 1990; Cameron et al., 1997). Since apoptosis has been reported in secretory endometrium (Hopwood and Levison, 1976; Kokawa et al., 1996) and bcl-2 immunoreactivity decreases in the secretory phase (Koh et al., 1995; Tao et al., 1997) steroids have been implicated in this process.

In the present study, endometrial tissue from healthy women exposed to antiprogestin in vivo in the early secretory phase was examined and the possible regulation of bcl-2 gene family members in the human endometrium by antiprogestin administration in vivo was considered. The objective was to investigate the hormonal control of apoptosis-related proteins by comparing the expression of bcl-2 and Bax in endometrium collected after the administration of mifepristone soon after ovulation to control mid-secretory endometrium.

\section{Materials and Methods}

A total of ten healthy women aged between 25 and 38 years (mean age 32) with regular menstrual cycles (25-35 days) were included in the study. The characteristics of the patients have been reported (Cameron et al., 1997). The mean body mass index of subjects was $25.5 \mathrm{~kg} \mathrm{~m}^{-2}$ (range 21.3-35.5). All subjects had a normal gynaecological examination. No subject had used hormonal contraception in the preceding 3 months and those women who were not surgically sterilized used a barrier method of contraception throughout the study period. The protocol was approved by the local Ethical Subcommittee and each woman provided written informed consent.

Subjects were studied over a control cycle and a treatment cycle. In the treatment cycle, subjects received $200 \mathrm{mg}$ mifepristone on day 2 after the onset of the LH surge in urine. The timing of the LH surge was detected by the subjects themselves using a commercially available home urine LH detection kit (Conceive; Quidel, San Diego, CA). Each women tested her urine twice a day at 08:00 $\mathrm{h}$ and 18:00 $\mathrm{h}$ from day 10 of the mentrual cycle (10 days after the start of menstruation) in both control and treatment cycles. Detection of the urine LH surge was confirmed by radioimmunoassays. An endometrial biopsy was collected with a pipelle suction curette (Prodimed, Neuilly-en-Thelle) from each subject 4 or 6 days after the LH surge on the same day of both control and treatment cycles. In the treatment cycle, the biopsy was thus taken 2 or 4 days after mifepristone ingestion.

All endometrial biopsies were fixed in $4 \%(\mathrm{w} / \mathrm{v})$ paraformaldehyde for histological assessment and immunohistochemical analysis. The histological appearances after haematoxylin and eosin staining have been reported (Cameron et al., 1997) and dated according to the criteria of Noyes et al. (1950).

\section{Immunohistochemistry}

Sections $(5 \mu \mathrm{m})$ of endometrial biopsies were examined for immunohistochemical localization of (i) the bcl-2 epitope using the technique of Koh et al. (1995), (ii) the Bax epitope, (iii) CD68-positive macrophages, (iv) CD56-positive large granular lymphocytes, and (v) the oestrogen receptor. Commercially available monoclonal and polyclonal antibodies were used for the detection of bcl-2 (DAKO Ltd, High Wycombe; Pezzella et al., 1990), Bax (Pharmingen, San Diego, CA), macrophages (DAKO, CD68 code M876), CD56-positive large granular lymphocytes (Zymed Laboratories, San Francisco, CA) and oestrogen receptors (DAKO, ER1D5).

\section{Pretreatment of sections for bcl-2, Bax, oestrogen receptor and CD56 immunohistochemistry}

Histoclear (National Diagnostics, Atlanta, GA) was used to remove the paraffin wax from the tissue sections, which were then rehydrated through a descending ethanol series and washed in $0.1 \mathrm{~mol}$ Tris-buffered saline (TBS) $\mathrm{l}^{-1}$ for bcl-2 and CD56, and $0.1 \mathrm{~mol} \mathrm{PBS}^{-1}$ for Bax and oestrogen receptor for $2 \times 5 \mathrm{~min}$. bcl-2, oestrogen receptor and CD56 epitopes were exposed by microwaving at high power in $0.01 \mathrm{~mol}$ sodium citrate buffer $1^{-1}, \mathrm{pH} 6.0$ for $2 \times 5$ min (oestrogen receptor), $3 \times 5 \mathrm{~min}$ (bcl-2) or $4 \times 5 \mathrm{~min}$ (CD56). The Bax epitope was exposed by microwaving at high power in distilled water for $2 \times 5 \mathrm{~min}$. After treatment, all sections were allowed to stand for $20 \mathrm{~min}$ in the microwave oven. After another wash in TBS or PBS, non-specific endogenous peroxidase activity was blocked by treatment with $3 \%(\mathrm{w} / \mathrm{v})$ hydrogen peroxide in distilled water for $10 \mathrm{~min}$ at room temperature. All tissue sections were exposed to a nonimmune block with either normal horse serum (for bcl-2, oestrogen receptor and CD56) or normal goat serum (for Bax) for $20 \mathrm{~min}$ at room temperature and then incubated with the appropriate primary antibody.

\section{Immunolocalization of $b c l-2$}

Tissue sections were incubated with monoclonal mouse anti-human bcl-2 antibody at a dilution of 1:40 for $60 \mathrm{~min}$ at $37^{\circ} \mathrm{C}$. Thereafter, tissue sections were labelled with an avidin-biotin peroxidase detection system (Vectastain; Vector Laboratories, Peterborough) that included the 
secondary biotinylated horse anti-mouse antibody. Tissue sections were then incubated with diaminobenzidine (DAB solution, DAB Kit; Vector Laboratories) for $10 \mathrm{~min}$. Finally, the sections were counterstained with haematoxylin, dehydrated, cleared in xylene and mounted in Pertex mounting medium (Cellpath plc, Hemel Hempstead). Negative controls were performed by replacing the primary antibodies with mouse immunoglobulin at the same concentration as the primary antibody.

\section{Immunolocalization of CD56}

Tissue sections were incubated with monoclonal mouse anti-human CD56 antibody at a dilution of 1:250 for $60 \mathrm{~min}$ at $37^{\circ} \mathrm{C}$ and labelled as described earlier.

\section{Immunolocalization of macrophages (CD68)}

Endometrial tissue sections were incubated with monoclonal mouse anti-human CD68 antibody at a dilution of 1:50 for $60 \mathrm{~min}$ at room temperature and labelled as described earlier. An antigen retrieval step was necessary to localize the CD68 epitope. Therefore, the tissue sections were subjected to an enzyme digestion in $0.1 \%(\mathrm{w} / \mathrm{v})$ trypsin and $0.1 \%(\mathrm{w} / \mathrm{v})$ calcium chloride, $\mathrm{pH}$ 7.8. The digestion was conducted at $37^{\circ} \mathrm{C}$ for $15 \mathrm{~min}$ and enzyme activity was then destroyed by washing the sections in tap water.

\section{Immunolocalization of Bax}

After pretreatment, tissue sections were incubated with polyclonal rabbit anti-human Bax antibody at a dilution of 1:1200 overnight at room temperature. Staining was again detected using an avidin-biotin peroxidase system. Negative controls were performed by replacing the primary antibody with non-immune rabbit immunoglobulin. First trimester villi provided a positive control and positive immunoreactivity was clearly visible in the cytotrophoblast layer (Lea et al., 1997a).

\section{Immunolocalization of oestrogen receptor}

After pretreatment, tissue sections were incubated with monoclonal mouse anti-human oestrogen receptor antibody at a dilution of $1: 25$ for $60 \mathrm{~min}$ at $37^{\circ} \mathrm{C}$. Thereafter, staining was detected using an avidin-biotin peroxidase system as described earlier.

\section{Data analysis}

Immunoreactivity was assessed using light microscopy and the intensity of immunostaining for bcl-2 and Bax protooncogenes in all tissue sections was scored semiquantitatively on an arbitrary four point scale, indicating the intensity and proportion of positively stained cells.
A score of 3 indicated intense staining of the majority of each cell type, that is, stromal and glandular epithelium or presumed leucocyte components. A score of 0 indicated an absence of staining and scores of 1 and 2 indicated weak or moderate intensity of immunoreactivity, respectively. Both the immunohistochemistry and the scoring of positively stained cells were performed by a single individual without prior knowledge of the phase of the menstrual cycle. Each subject in the study underwent a single endometrial biopsy in each of the control and treatment cycles. Duplicate sections were analysed for each biopsy. One section was exposed to the primary antibody and the other section served as the negative control. Immunostaining in the whole tissue section was taken into consideration in the assessment; at least ten glands were evaluated per section. Immunoreactivity was fairly homogenous for bcl-2, Bax and oestrogen receptor. Immunohistochemistry is associated with variability in immunostaining and hence each antibody was applied to each series of tissue sections in a single run. It is important to note that the immunolocalization of CD56- and CD68positive leucocytes was undertaken to identify the characteristics of the presumed leucocyte subtype with positive immunostaining of the bcl-2 epitope. Serial sections were used for this purpose.

ANOVA was used to evaluate whether there was any significant difference in immunoreactivity recorded in the semi-quantitative system described earlier. Assignment of significance of differences was determined by Fisher's protected least significant difference test. Correlations were derived from, and correlation matrix was obtained using, Statview 4.5 .

\section{Results}

In control endometrium in the secretory phase on days 4 and 6 , mild positive immunoreactivity for bcl- 2 was observed in both the glandular and surface epithelium (Figs 1a and 2a). There was a significant increase in positive immunoreactivity $(P<0.01)$ in glandular and surface epithelium after administration of mifepristone (Figs $1 \mathrm{a}$ and $2 b)$. There was no significant change in bcl-2 stromal immunoreactivity in endometrial biopsies collected on day 4 or day 6 after the LH surge and in the treatment cycle after administration of mifepristone (Fig. 1a).

Isolated stromal cells showed positive immunoreactivity for the bcl-2 epitope. However, there was no significant change in immunostaining in these stromal cells before and after administration of mifepristone. Examination of serial sections with the cell surface markers CD68 and CD56 indicated that the isolated stromal cells were likely to be either CD68-positive macrophages or CD56-positive large granular lymphocytes (Fig. 3).

Low immunoreactivity for the Bax epitope was observed in glandular and surface epithelium in all control biopsies (collected on days 4 and 6 after the LH surge) (Fig. 2c). There was no significant change in positive immunostaining after administration of mifepristone (Figs $1 \mathrm{~b}$ and 2d). Positive immunoreactivity was also noted in stromal tissues in both 

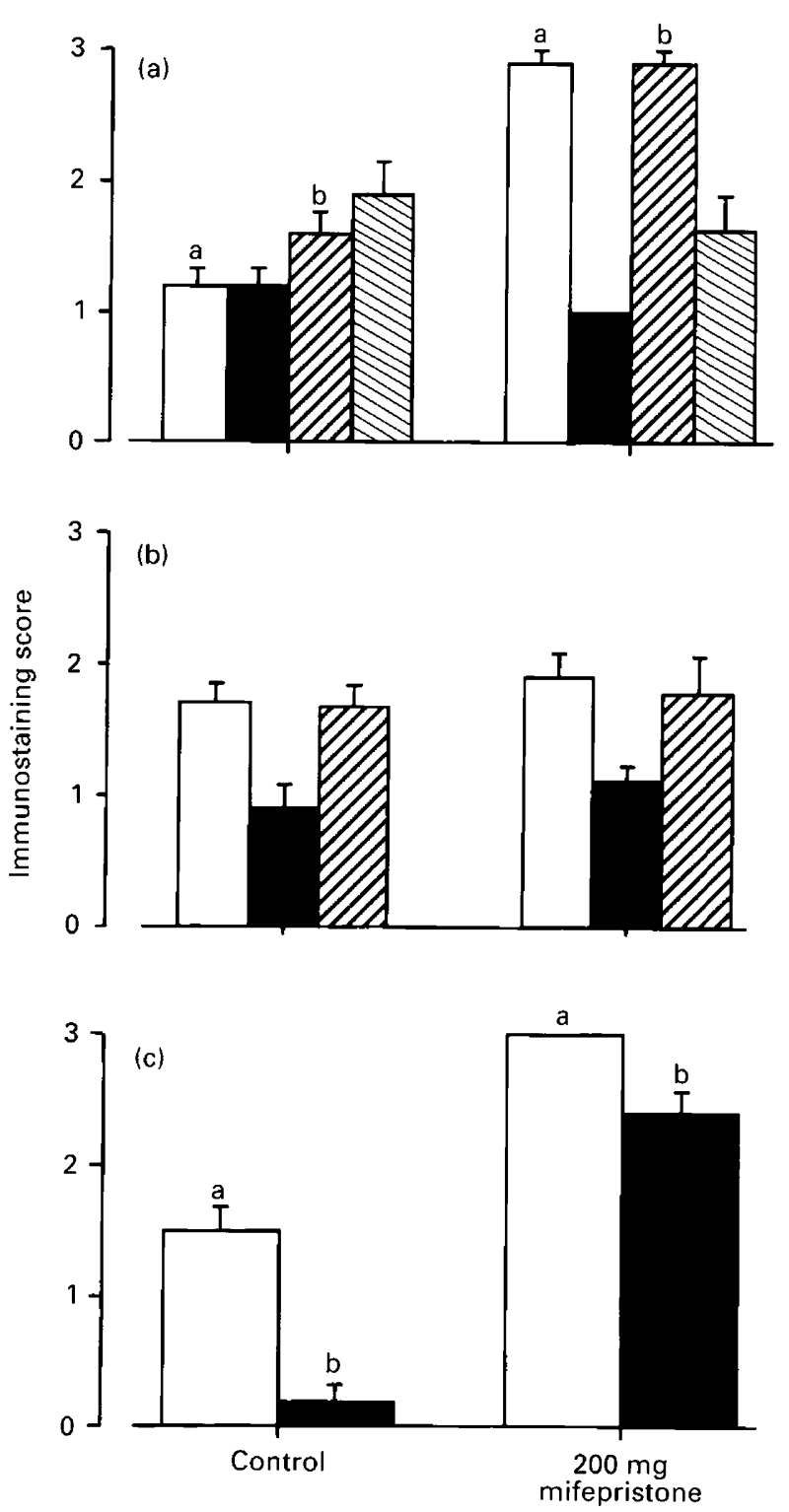

Fig. 1. Immunostaining scores for (a) bcl-2, (b) Bax and (c) oestrogen receptor in human endometrium with and without administration of mifepristone on day 2 after the onset of the LH surge. $\square$,

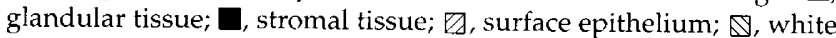
blood cells. Endometrial biopsies for control and treated groups were collected on day 4 or day 6 after the LH surge. Values are mean \pm SEM.

the control and treated endometrium (Figs $1 \mathrm{~b}$ and $2 \mathrm{c}, \mathrm{d}$ ), but this was less pronounced than in the glandular and surface epithelium.

A significant increase in oestrogen receptor immunoreactivity was observed in both the glandular and stromal endometrial tissue after administration of antiprogestin (Fig. 1c). A positive correlation (0.874) was demonstrated between bcl-2 immunoreactivity in glandular tissue and the positive nuclear immunoreactivity for glandular oestrogen receptors.

\section{Discussion}

The results of the present study confirm that bcl-2 and Bax proteins are localized mainly to the glandular and surface epithelium of secretory endometrium. After administration of the antiprogestin mifepristone in the early luteal phase, the development of secretory changes in the endometrium was inhibited (Swahn et al., 1990; Cameron et al., 1997). Antiprogestins inhibit the expression of a number of progesterone-dependent proteins, including prostaglandin dehydrogenase, glycodelin, $17 \beta$ steroid dehydrogenase and leukaemia inhibitory factor. In addition, the downregulation of oestrogen and progesterone receptors that normally occurs during the luteal phase is prevented (Cameron et al., 1997). The increase in immunostaining for bcl-2 protein in the glands of women administered mifepristone indicates that the expression of this gene may be stimulated by oestrogen and downregulated by progesterone.

An increase in bcl-2 concentrations in glandular tissue would decrease apoptosis and extend the life span of the epithelial cells. Since an increase in survival of epithelial cells in the colon has been associated with an increased risk of carcinoma (Tsujii and DuBois, 1995; Tsujii et al., 1998), the increase in bcl-2 after treatment with antiprogestin reinforces the protective role of progesterone in an oestrogendominated uterus.

The isolated cells in the stromal compartment that stained strongly for bcl-2 also showed clear staining for CD68 and CD56, indicating that these cells may be subpopulations of macrophages or large granular lymphocytes. The fact that the degree of staining in the cells was not influenced by administration of mifepristone indicates that they are not under the influence of progesterone.

Oestrogen induces proliferation and growth of glandular and stromal cells by interacting with oestrogen receptors. It is thought that the anti-proliferative effect of progesterone is due to its ability to downregulate expression of the oestrogen receptor. Mifepristone prevents this action of progesterone and hence a large number of oestrogen receptors (and progesterone receptors) persists in the endometrium of women administered this compound in the early luteal phase (Cameron et al., 1997). It is possible that the upregulation of $\mathrm{bcl}-2$ that occurs after treatment with mifepristone is due to the persistence of oestrogen receptors. However, in spite of the high concentration of oestrogen receptors and a marked increase in Ki67 expression (Cameron et al., 1996), the endometrium shows little or no evidence of mitosis after administration of mifepristone. The anti-proliferative action of mifepristone is not fully understood, but is thought to be due to a product of the mifepristone-progesterone receptor complex that prevents the cells from completing the $\mathrm{S}$ phase of the cell cycle (Cameron et al., 1996).

The immunoreactivity of the Bax epitope was constant both in control endometrium and in endometrium after administration of antiprogestin. This is consistent with the observations of McLaren et al. (1997) and Tao et al. (1997) of Bax immunostaining in glandular epithelium. McLaren et al. (1997) indicated that this finding implies a lack of hormone regulation of this epitope. The observations of this group are 

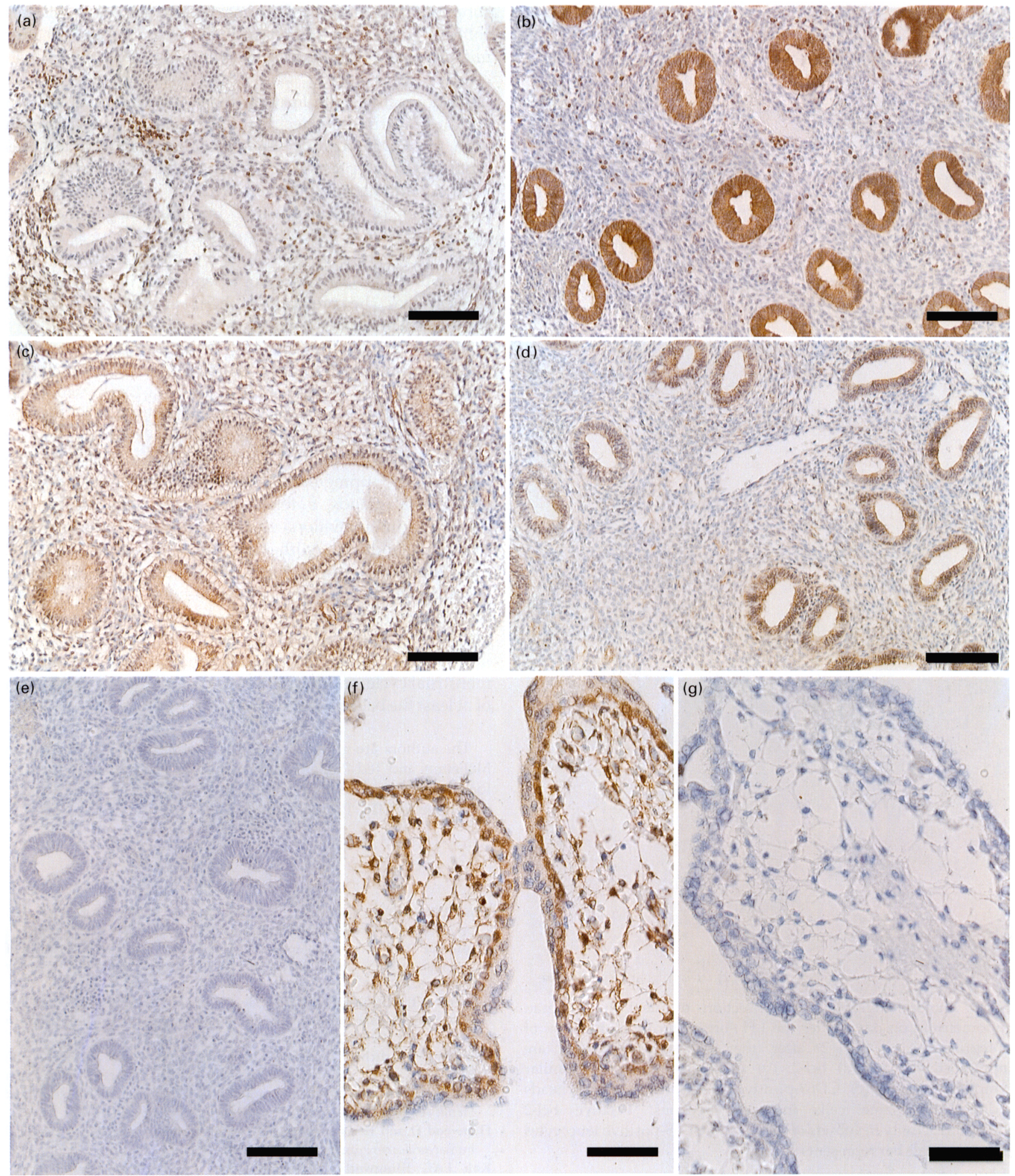

Fig. 2. bcl-2 immunoreactivity in luteal phase human endometrium on day 4 after the LH surge (a) without and (b) with administration of mifepristone on day 2 after the LH surge. Bax immunoreactivity in luteal phase human endometrium (c) without and (d) with administration of mifepristone on day 2 after the LH surge; results from (c) day 6 and (d) day 4 after the LH surge. (e) Negative control, bcl-2 primary antibody replaced with mouse immunoglobulin at equivalent concentration. (f) Positive control for Bax immunostaining, first trimester villi with positive immunoreactivity (brown) in the cytotrophoblast; (g) negative control for Bax immunoreactivity, first trimester villi absent immunoreactivity. Scale bars represent $(\mathrm{a}-\mathrm{e}) 100 \mu \mathrm{m},(\mathrm{f}, \mathrm{g}) 50 \mu \mathrm{m}$. 

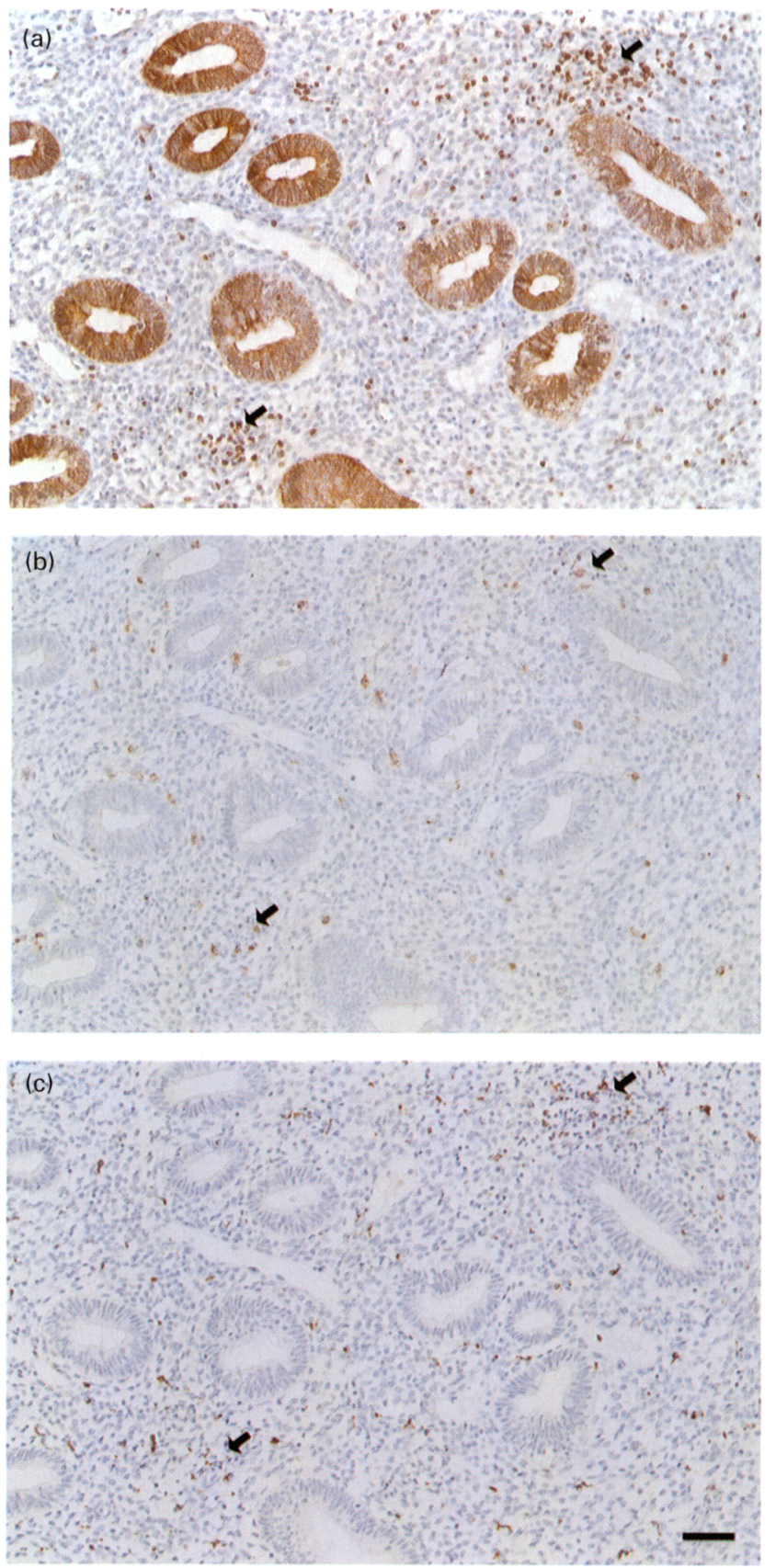

Fig. 3. Photomicrographs of serial sections of luteal phase human endometrium on day 4 after the LH surge after administration of mifepristone on day 2 after the LH surge. Sections are immunostained for (a) bcl-2; (b) CD56-positive large granular lymphocytes and (c) CD68-positive macrophages. Arrowheads indicate the same cells in serial sections. Positive bcl-2 immunoreactivity in subsets of CD56- and CD68-positive leucocytes is shown. Scale bar represents $50 \mu \mathrm{m}$.

in contrast to those of Tao et al. (1997), since the latter report does consider Bax expression to be hormone regulated. In the present study, there was no difference in Bax immunostaining between control endometrium and tissues collected after mifepristone administration.

The observation of bcl-2 positive immunoreactivity in a subpopulation of isolated stromal cells in the control tissues collected in the secretory phase and in the endometrium after mifepristone administration is of interest. This group (Koh et al., 1995; Lea et al., 1997b) has identified bcl-2 immunopositive cells in non-pregnant endometrial stroma and in first trimester decidua. Furthermore, co-localization with the CD56-positive large granular lymphocytes was reported. In the present study macrophages were observed in the endometrial biopsies. This is consistent with a population of bcl-2 positive but Bax negative tissue macrophages in ectopic endometrial tissues (McLaren et al., 1997). McLaren et al. (1997) report the presence of such subpopulations only in ectopic endometrial tissues, and suggest that the presence of bcl-2 and absence of Bax contributes to the survival of the macrophages.

In conclusion, the findings of the present study in antiprogestin-treated human endometrium support earlier reports of steroid regulation of at least bcl-2 expression and possible modulation via the oestrogen receptor (Otsuki et al., 1994; Teixeira et al., 1995). Blocking the progesterone receptor with mifepristone on day 2 after the onset of the LH surge resulted in upregulation of oestrogen receptors in endometrial glands, a retardation of the development of secretory differentiation, and continued bcl- 2 expression in the glandular epithelium. The presence of bcl-2 immunoreactivity in a subset of macrophages and large granular lymphocytes indicates continued survival of these leucocyte subpopulations in endometrium after antiprogestin exposure. These observations are consistent with morphological changes in the endometrium throughout the menstrual cycle, and support steroid regulation of expression of at least the bcl-2 protein in the uterine epithelium.

The authors are grateful for the assistance of Stuart Milne, Tom McFetters and Ted Pinner with illustrations in this manuscript and for the secretarial assistance of Vicky Watters. This research was supported by MRC Project No. G9620138.

\section{References}

Baird DT (1993) Antigestogens. In British Medical Bulletin 49 Contraception pp 73-87 Eds DT Baird and JO Drife. Churchill Livingstone, Edinburgh

Baulieu EE (1997) RU486 (Mifepristone) a short overview of its mechanism of action and clinical uses at the end of 1996 Annals of the New York Academy of Sciences 828 47-58

Cameron ST, Critchley HOD, Buckley CH et al. (1996) Effects of daily low dose mifepristone on endometrial maturation and proliferation Human Reproduction 11 2518-2526

Cameron ST, Critchley HOD, Buckley CH, Kelly RW and Baird D (1997) Effects of two antiprogestins (mifepristone and onapristone) on endometrial factors of potential importance for implantation Fertility and Sterility 67 1046-1053

Hopwood D and Levison DA (1976) Atrophy and apoptosis in the cyclical human endometrium Journal of Pathology 119 159-165

Koh EAT, Illingworth PJ, Duncan WC and Critchley HOD (1995) Immunolocalisation of bcl-2 protein in human endometrium in the menstrual cycle and simulated early pregnancy. Molecular Human Reproduction 1 Human Reproduction 10 1557-1562

Kokawa K, Shikone T and Nakano R (1996) Apoptosis in the human uterine endometrium during the menstrual cycle journal of Clinical Endocrinology and Metabolism 81 4144-4147

Lea RG, Riley SC, Tulppala M and Critchley HOD (1997a) Placental apoptosis and pregnancy failure Journal of Reproduction and Fertility Abstract Series 20 Abstract 3 
Lea RG, Al-Sharekh N, Tulppala M and Critchley HOD (1997b) The immunolocalisation of $\mathrm{bcl}-2$ at the maternal-fetal interface in healthy and failing pregnancies Human Reproduction 12 153-158

McLaren J, Prentice A, Charnock-Jones DS, Sharkey AM and Smith SK (1997) Immunolocalisation of the apoptosis regulating proteins bcl-2 and Bax in human endometrium and isolated peritoneal fluid macrophages in endometriosis Human Reproduction 12 146-152

Noyes RW, Hertig AT and Rock J (1950) Dating the endometrial biopsy Fertility and Sterility 1 3-25

Oltavi ZN, Milliman CL and Korsmeyer SJ (1993) bcl-2 heterodimerises in vivo with a conserved homologue Bax that accelerates programmed cell death Cell 74 609-619

Otsuki Y, Misaki O, Sugimoto O, Ho Y, Tsujimoto $Y$ and Akao Y (1994) Cyclic bcl-2 gene expression in human uterine endometrium during menstrual cycle Lancet 344 28-29

Pezzella F, Tse AGD, Cordell JL, Pulford KAF, Gatter KC and Mason DY (1990) Expression of the bcl-2 oncogene protein is not specific for the 14-18 chromosomal translocation American Journal of Pathology 137 225-232

Swahn M-L, Byedeman M, Cekan S, Xing S, Masironi B and Johanisson E (1990) The effect of RU486 administered during the early luteal phase on bleeding, hormonal parameters and endometrium Human Reproduction $402-408$

Tao X-J, Tilly KI, Maravei DV, Shifren JL, Krajewski S, Reed JC, Tilly JL and Isaacson KB (1997) Differential expression of members of the bcl-2 gene family in proliferative and secretory human endometrium: glandular epithelial cell apoptosis is associated with increased expression of bax Journal of Clinical Endocrinology and Metabolism 82 2738-2746

Teixeira C, Reed JC and Pratt MAC (1995) Estrogen promotes chemo therapeutic drug resistance by a mechanism involving bcl-2 proto-oncogene expression in human breast cancer cells Cancer Research 55 3902-3907

Tsujii M and DuBois RN (1995) Alterations in cellular adhesion and apoptosis in epithelial cells overexpressing prostaglandin endoperoxide synthesase 2 Cell 83 493-501

Tsujii M, Kawano S, Tsuji S, Takei Y, Nagai H, Sawaoka H, Murata H, Kawai N, Hori $M$ and DubBois RN (1998) Effect of cyclooxygenase-2 expression in colon cancer cells on chemotaxis of neutrophils and macrophages Gastroenterology 114 G2867

Wyllie AH (1994) Death gets a break Nature (London) 369 272-273

Wyllie AH, Kerr JFR and Currie AR (1980) Cell death: the significance of apoptosis International Review of Cytology 68 251-306 